\title{
Resistance and virulence properties of extraintestinal pathogenic $E$. coli causing nosocomial-and community-acquired urinary tract infections in hospitalized patients in Rio de Janeiro, Brazil
}

Ana Carolina da C. Campos ${ }^{1,2}$, Nathália Andrade ${ }^{2}$, Julio C. D. Correal ${ }^{3}$, Carla C. Santos ${ }^{3}$, Marines du Teil Espina1, Jan Maarten van Dij1 ${ }^{1}$, Monika A. Chlebowicz-Fliss ${ }^{1}$, Alex W. Friedrich ${ }^{1}$, Nico T. Mutters $^{4}$, Paulo V. Damasco ${ }^{5,6}$, Ana Cláudia de P. Rosa ${ }^{2}$ and John W. A. Rossen ${ }^{1,7,8, *}$

1 University of Groningen, University Medical Center Groningen, Department of Medical Microbiology and Infection prevention, The Netherlands.; anabio86@gmail.com; alex.friedrich@umcg.nl; john.rossen@gmail.com; monikachlebowicz@gmail.com; j.m.van.dij101@umcg.nl; m.du.teil.espina@umcg.nl

2 Universidade do Estado do Rio de Janeiro, Faculdade de Ciências Médicas, Departamento de Microbiologia, Imunologia e Parasitologia, Brazil.; nathalu84@yahoo.com.br; paulovieiradamasco@gmail.com; anarosa2004@gmail.com

3 Hospital Rio Laranajeiras, Brazil; jucedelco@hotmail.com; ccih@fblo.com.br

4 Bonn University Hospital, Institute for Hygiene and Public Health, Germany; nico.mutters@ukbonn.de

5 Universidade do Estado do Rio de Janeiro, Departamento de Doenças Infecciosas e Parasitárias, Brazil.

6 Universidade Federal do Rio de Janeiro, Departamento de Doenças Infecciosas e Parasitárias, Brazil.

7 University of Utah, School of Medicine, Department of Pathology, UT, USA.

8 IDbyDNA Inc., Salt Lake City, UT, USA.

* Correspondence: John W.A. Rossen. Department and Address: Department of Medical Microbiology and Infection Prevention, University of Groningen, University Medical Center Groningen. EB80 Hanzeplein 1, 9713 GZ, Groningen, The Netherlands. Tel: +1 650319 8838. E-mail: john.rossen@gmail.com

Received: date; Accepted: date; Published: date

\begin{abstract}
Background: Extraintestinal pathogenic Escherichia coli (ExPEC) is the most common cause of urinary tract infections (UTIs). They are often multidrug-resistant (MDR), making them challenging to treat. Additionally, virulence mechanisms as biofilm formation are associated with persistent UTIs. Aims: To reveal a possible association between patients' risk factors and UTIs caused by MDR or biofilm-forming ExPECs and characterize ExPECs causing asymptomatic bacteriuria, community- (CA), or hospital-acquired (HA) UTIs in hospitalized patients in Brazil. Methods: Bacterial DNA was extracted from the urine of 63 hospitalized patients and sequenced using short-read sequencing. Antibiotic susceptibility was evaluated using VITEK-2, and the biofilm-forming, adhesion, and invasion abilities were quantitatively assessed. Results: Antibiotic resistance rates were high, and the majority of UTIs were complicated CA-UTIs. Most MDR- and ESBL-producing E. coli isolates belonged to high-risk lineages and were associated with UTIs in patients with comorbidities and over 60 years of age. The mortality rate of patients infected with MDR-isolates was higher than of those infected with non-MDR isolates. Most isolates were biofilmforming, but no association with patients' risk factors was found. Conclusions: Complicated UTIs caused by MDR- and biofilm-forming bacteria are frequently found in hospitalized patients in Brazil suffering from a UTI and are associated with high-risk lineages.
\end{abstract}

Keywords: urinary tract infections; multi-drug resistance; biofilm; virulence genes; WGS. 


\section{Introduction}

Extraintestinal pathogenic Escherichia coli (ExPEC), especially uropathogenic E. coli (UPEC), are the most common cause of hospital- (HA) and community-acquired (CA) urinary tract infections (UTIs) [1]. Besides, they are also the primary cause of asymptomatic bacteriuria (ABU). With the emergence of multi-drug resistant (MDR) E. coli and the slow development of new antibiotics, treatment options are often scarce [2]. Antimicrobial resistance contributes to bacterial persistence and can lead to chronic and more severe infections [3]. Another factor determining the severity of infections caused by $E$. coli is the presence of virulence factors that play an important role in the initial phase of infection by allowing the adhesion and colonization of host mucosal surfaces [4]. Also, biofilm formation increases an isolate's virulence as it helps bacteria to persist in the genitourinary tract and interferes with bacterial eradication [5]. Remarkably, UPEC can form an intracellular biofilm-like pod in urogenital cells, the so-called complex intracellular bacterial community, and form biofilms on indwelling medical devices [6], leading to device-associated infections.

The combination of high resistance and virulence profiles in E. coli contributed to the booming global spread of these bacteria. UTIs can be classified into uncomplicated and complicated infections. The latter is associated with the presence of a patient's risk factors [7, 8]. In Brazil, the increase of UTIs caused by MDR E. coli represents a severe public health problem. Few studies have focused on a possible association of the patient's risk factors and the resistance and virulence characteristics of $E$. coli. Such information is crucial as it helps to improve the risk assessment of and treatment strategies against UTIs. Therefore, we aimed to reveal the characteristics, including the biofilm-forming ability, adhesion and invasion abilities, antibiotic susceptibility, the presence of virulence and resistance genes, and the sequence type (ST) of E. coli isolated from patients with ABU and UTIs hospitalized in Rio de Janeiro, Brazil, and their possible association with patients' risk factors.

\section{Results and Discussion}

This study characterized 63 E. coli isolates from patients with ABU and UTIs admitted to hospitals in Rio de Janeiro, Brazil. Most of the isolates were from female patients $(\mathrm{n}=46 ; 73 \%)$ and patients older than 60 years (mean age $67.9 \pm 3.3$ years old) (Table 1). These results agree with previous studies showing that UTIs are more frequently found in women and older adults [2]. The most frequently identified patient's risk factors were neurologic and neoplastic diseases, diabetes, previous hospitalization, surgical procedures, obstructive diseases of the urinary tract, and catheterization (Table 1). Our findings are consistent with previous findings that hospitalization, surgical procedures, and comorbidities were associated with UTIs in hospitalized patients [16][8]. Although the frequency of CA-UTIs was higher than that of HA-UTIs, this difference was not statistically significant. In addition, the HA-UTIs cases were more identified among male patients (64\%). As shown by others, HA-UTIs in males often resulted in complicated UTIs [17].

MDR-E. coli, mainly ESBL-producing ones, are of increasing clinical concern due to the limited antibiotic options to treat these infections [18]. In our study, $33(52.3 \%)$ patients were infected with MDR E. coli and 25 (39.6\%) with ESBL-producing ones. Several studies have investigated patients' risk factors associated with UTIs, especially those caused by MDR bacteria [19]. Here, most of the MDR E. coli were isolated from patients also suffering from comorbidities, mainly diabetes $(\mathrm{OR}=3.913$; 95\%CI 0.960-15.947; $\mathrm{p}=0.045)$, neurologic diseases ( $\mathrm{OR}=3.941 ; 95 \% \mathrm{CI} 1.177-10.354 ; \mathrm{p}=0.020)$ and neoplastic diseases (OR=3.913; 95\%CI 0.960-15.947; $\mathrm{p}=0.045)$. Indeed, diabetes and neurologic diseases have been pointed as risk factors for UTIs in hospitalized patients before [20]. UTIs caused by ESBL-producing bacteria were also associated with an age older than 60 years (OR=3.580; 95\%CI 1.021-12.551; $\mathrm{p}=0.036)$, consistent with previous results showing age to be an independent risk factor for UTIs caused by ESBL-producing isolates [21]. The association between neoplastic diseases and MDR infections may be explained by the immunosuppressed condition of patients with neoplastic 
diseases caused by the treatment of their disease. Indeed, immunosuppression is a risk factor for the emergence of MDR uropathogens [22].

Bacterial biofilm is often associated with the persistence of microorganisms in the host and with catheter-associated UTIs [5]. Therefore, revealing the biofilm-forming ability of isolates is of clinical relevance. In our study, most isolates were able to form biofilm (Figure 1). These results are consistent with previous studies that showed that about $80 \%$ of $E$. coli isolated from urine samples produced biofilms [23]. We investigated if biofilm-forming isolates were associated with patients having comorbidities and other risk factors for UTIs. However, no statically significant association was found. In addition, we investigated the presence of virulence genes associated with ABU, CA- and HA-UTIs. The distribution of virulence genes of the isolates obtained from patients with an HA- or CA-UTIs or with ABU were similar, except for the vat and usp genes that were more frequently identified in CA-UTIs isolates. Our results may be explained by the predominance of isolates that belong to the B2 and D phylogenetic groups in our study, which are known to be more virulent. We did not find an association between the presence of virulence genes and either MDR or non-MDR bacterial isolates, although the number of virulence genes was generally higher in MDR isolates. This may be explained by the fact that in ExPEC isolates, many virulence genes are located on mobile genetic elements, such as plasmids, pathogenicity islands, and bacteriophages, which can easily be exchanged between strains. Moreover, they are often required and/or beneficial for both MDR and non-MDR bacteria to persist in the gut [24].

The worldwide increase of infections caused by MDR bacteria has been associated with the spread of specific bacterial lineages that are CTX-M $\beta$-lactamase-producing and fluoroquinolones resistant [25]. Our previous study showed that E. coli with ST10, ST69, ST131, ST405, and ST648 were the most prevalent STs causing UTIs found in the investigated hospitals. Here, ST131 was more frequently found in CA-UTIs. Furthermore, MDR and ESBL-producing isolates belonging to ST131, ST405, and ST648 were responsible for approximately 35\% of the CA-and HA-UTIs. The predominance of ST131, ST405, and ST648 have been described previously [26]. Considering the high number of UTIs caused by high-risk lineages (ST131, ST405, and ST648) in this study, we investigated the virulence characteristics of this group and the patients' risk factors from which they were isolated. The results were compared with data obtained for non-high-risk lineages frequently identified in this study. Overall, UTIs caused by high-risk clones were more frequently detected in elderly patients (86.3\%), patients suffering from neurologic diseases (50\%), and in patients with complicated infections (Table 2). These results may be (partially) explained by the higher rate of previous hospitalization and antibiotic treatment in elderly patients and patients with neurologic diseases. Indeed, previous studies considered hospitalization and previous antibiotic treatment as host risk-factors associated with infections caused by E. coli high-risk clones as ST131 [22,27]. The mortality rate was found to be higher for patients suffering from UTIs caused by MDR or ESBL-producing isolates. The same association was found for UTIs caused by high-risk clones. These results may be explained by the known association between high-risk clones and an MDR/ESBL profile. The limited antibiotic treatment options and the presence of several risk-factors often present in patients having a UTI caused by a high-risk clone, which increases their susceptibility to infections, may explain this higher mortality rate. Nevertheless, this study has several limitations, including the relatively small sample size. Secondly, information about the severity of infections was not available for all patients but may have had an important impact on the correlation between risk factors and isolates characteristics and/or the mortality rate. When we investigated the adhesion and invasion abilities, ST131 isolates showed the same ability to adhere to uroepithelial cells as other lineages. However, ST69 and ST1703 isolates had a higher ability to invade cells (Figure 2). These results showed that despite the high-risk clones being statistically associated with the presence of the afa and iha genes encoding fimbrial adhesins and the iron-regulated adhesin, respectively, the high-risk clones presented a similar adhesion ability as non-high-risk clones. However, the non-high-risk clones presented a better invasion ability. Although, this difference may be isolate-specific rather than being related to a specific lineage. 


\section{Quantitative Biofilm Formation}

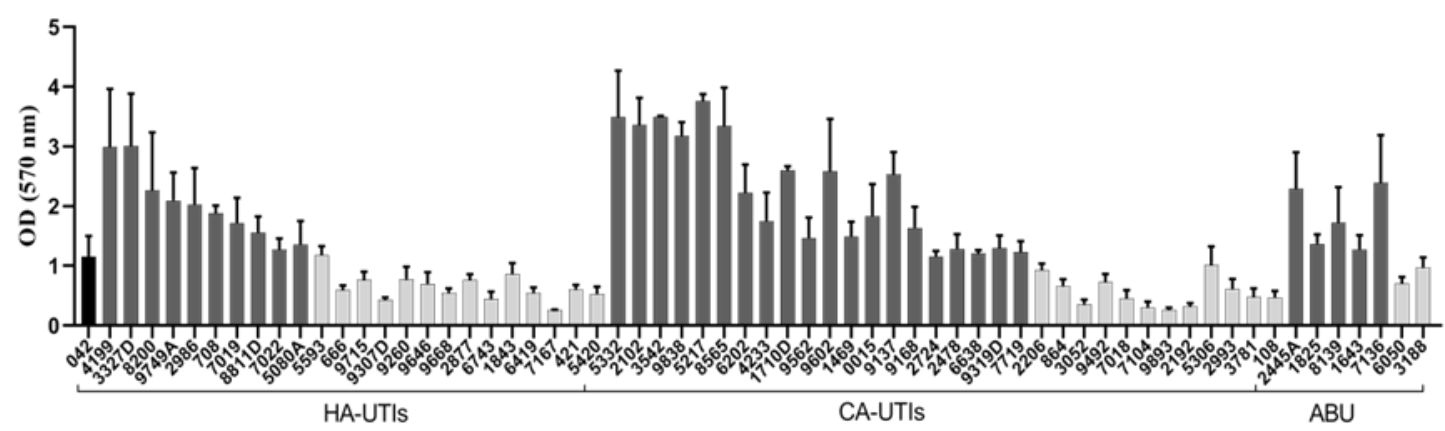

Figure 1. Quantitative biofilm-forming results of E. coli isolates. The isolates were divided into nonadherent (light grey bars), and biofilm-forming (dark grey bars) isolates based on results obtained for the control strain EAEC 042 (black bar). Error bars indicate variation between the replicates (duplicates from three independent experiments).

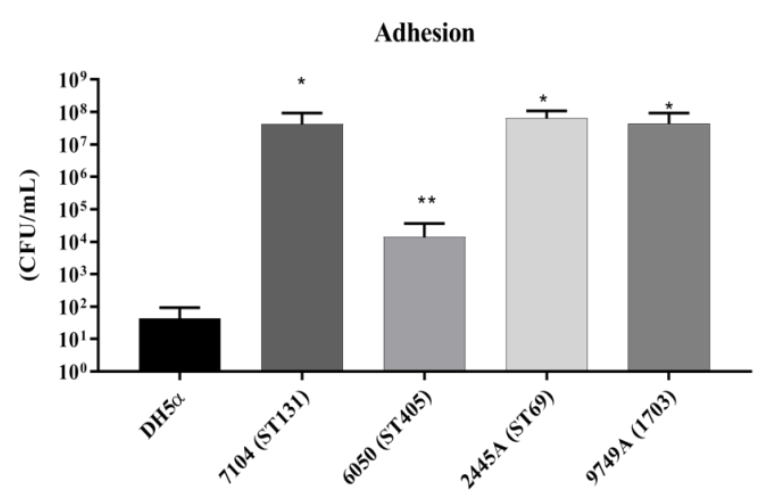

(a)

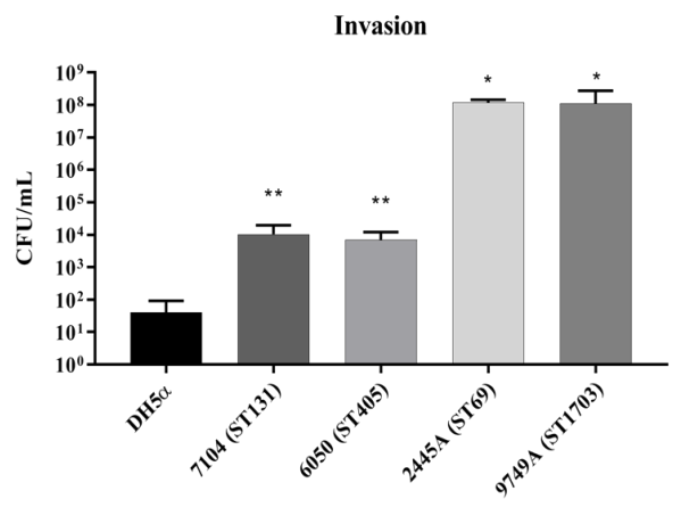

(b)

Figure 2. Adhesion and Invasion of E. coli isolates. (a) the results of the adhesion assay using representative isolates of ST131, ST405, ST69, and ST1703. (b) the results of the invasion assay with the same isolates. The black bar indicates the DH5 $\alpha$ strain used as a negative control, and the greycolored bars indicate the study isolates. ${ }^{*}$ indicates a statistically significant difference $(\mathrm{p}<0.05)$ compared to the negative control, and ${ }^{* *}$ indicates a statistically significant difference $(\mathrm{p}<0.05)$ compared to the negative control and the other isolates. Using the averages of each CFU/mL count using three biological replicates. 
Table 1. Characteristics of patients included in this study.

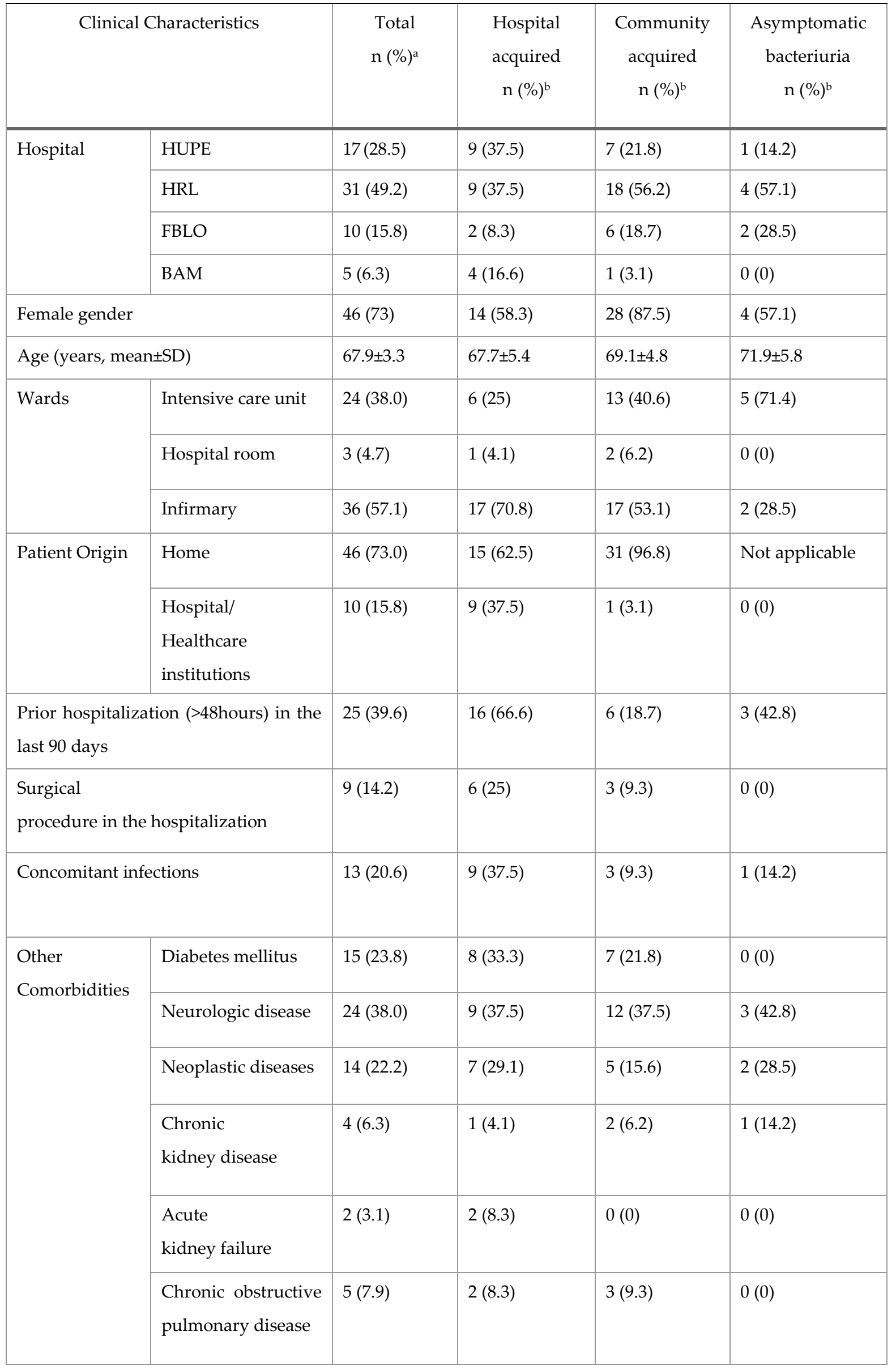




\begin{tabular}{|l|l|l|l|l|l|}
\hline & $\begin{array}{l}\text { Obstructive } \\
\text { pathology of the } \\
\text { urinary tract }\end{array}$ & $9(14.2)$ & $3(20.8)$ & $1(14.2)$ \\
\hline $\begin{array}{l}\text { Other } \\
\text { risk factors to } \\
\text { UTIs }\end{array}$ & Indwelling catheter & $5(7.9)$ & $2(8.3)$ & $2(6.2)$ & $1(14.2)$ \\
\cline { 2 - 6 } & $\begin{array}{l}\text { Immunosuppressor } \\
\text { use }\end{array}$ & $3(4.7)$ & $2(8.3)$ & $0(0)$ & $1(14.2)$ \\
\hline HIV & $1(1.5)$ & $0(0)$ & $0(0)$ & $1(14.2)$ \\
\hline Trauma & $9(14.2)$ & $5(20.8)$ & $3(9.3)$ & $1(14.2)$ \\
\hline Mortality rate & $17(26.9)$ & $7(29.1)$ & $7(21.8)$ & $3(42.8)$ \\
\hline Complicate infections & $46(73.0)$ & $22(91.6)$ & $24(75)$ & Not applicable \\
\hline Biofilm-forming & $50(79.3)$ & $18(75)$ & $25(78.1)$ & $7(100)$ \\
\hline
\end{tabular}

${ }^{a}$ Percentages were based on the total number of patients $(n=63) ;{ }^{b}$ Percentages were based on the total number of patients for the indicated type of infection. HUPE, HUPE-University Hospital Pedro Ernesto; HRL, RioLaranjeiras Hospital. FBLO, Bela Lopes de Oliveira Foundation; BAM, Bambina Hospital. All hospitals are located in Rio de Janeiro, Brazil. 
Table 2. Characteristics of E. coli isolates causing HA-, CA-UTIs or ABUs.

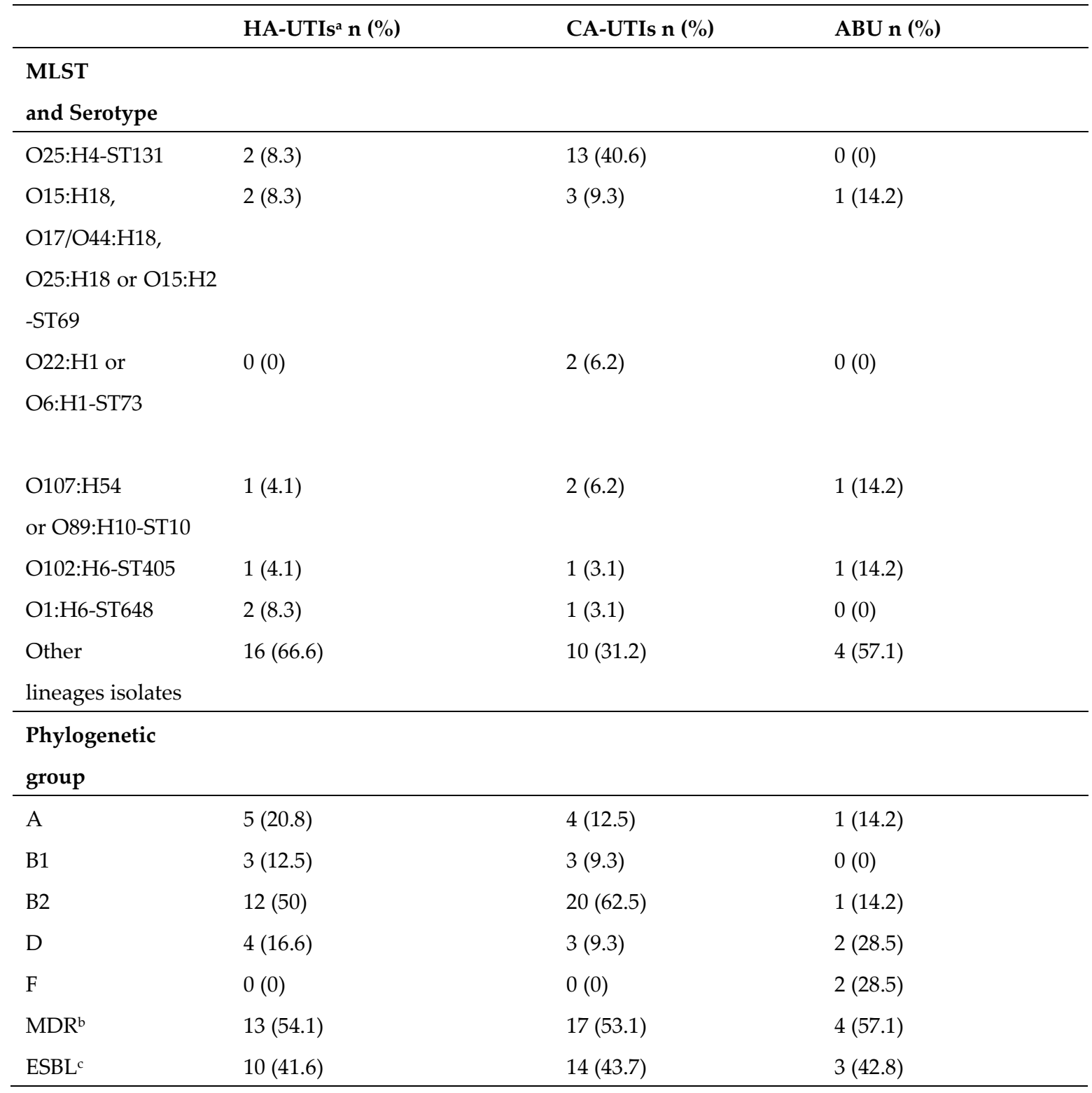

aHA-UTIs: hospital acquired urinary tract infections (UTIs); CA-UTIs: community acquired UTIs; ABU: asymptomatic bacteriuria. ${ }^{b} \mathrm{MDR}$ : multidrug resistance. ${ }^{\mathrm{c} E S B L}$ : extended-spectrum-beta-lactamase.

\section{Materials and Methods}

\subsection{Bacterial strains, growth conditions, antimicrobial susceptibility and case definitions}

A total of 63 previously collected E. coli isolates [9] were used in this study. The well-characterized strain 042 was used as a positive control for biofilm formation [10]. Susceptibility tests were performed using the Vitek-2 (bioMérieux, Marcy l'Etoile, France) and interpreted following EUCAST guidelines (v7.1, 2017). Isolates resistant to three or more antimicrobial classes were considered to be MDR. Data from patients suffering from ABU or CA- or HA-UTIs were collected, including age, sex, mortality rates (frequency of occurrence of death among the patients in our study between November 2015-November 2016), and risk factors as, e.g., primary site of infection, comorbidities, use of immunosuppressors and previous hospitalization. All patient's strains from located and interviewed patients were included in this study. The patients were interviewed, and their medical records were 
reviewed to obtain demographic and clinical data. ABU were defined as cases where $10^{5} \mathrm{CFU} / \mathrm{mL}$ of E. coli was isolated from urine samples of patients without any symptoms of a UTI. Comorbidities were defined as one or more coexisting medical conditions along with the diagnosis of UTIs or ABU. HA-UTIs were defined as cases where the urine samples were collected at least $72 \mathrm{~h}$ after admission to the hospital, and the patient did not have clinical symptoms before or during this period. Complicated infections were defined as UTIs occurring in a host with predisposing conditions (riskfactors), regardless of the presenting syndrome or severity of illness.

\subsection{DNA extraction, whole genome sequencing and molecular analysis}

Total DNA was extracted from all 63 isolates using the Ultraclean ${ }^{\circledR}$ microbial DNA isolation kit (Qiagen). A DNA library was prepared using the Illumina Nextera XT kit, and libraries were sequenced on a MiSeq (Illumina) as previously described [9]. De novo assembly was performed using CLC Genomics Workbench v10.0.1 (Qiagen, CLC bio A/S, 143 Aarhus, Denmark). Annotation was performed by uploading sequences to the RAST server version 2.0 [11]. The multi-locus sequence types (MLST) and whole-genome (wg) MLST complex types (CTs) were identified by uploading assembled genomes to SeqSphere v4.1.9 (Ridom, Muster, Germany). For the wgMLST analysis in seqsphere, a 2764 genes core genome (cg) scheme combined with 1785 accessory genes was used [12]. Resistance genes were identified by uploading assembled genomes in fasta format to ResFinder (CGE) [13]. Virulence genes were identified by uploading assembled genomes in fasta format to VirulenceFinder 1.5 [14]. Other specific virulence genes were identified by blasting assembled genomes against known virulence reference genes downloaded from NCBI or the ENA database using CLC Genomics Workbench v10.0.1 (Qiagen, CLC bio A/S, Aarhus, Denmark). These genes include iron uptake system genes (iha, iroN, fyuA, fhuA, fhuE, irp2, iutA), toxin genes (cnf1, hlyA, hlyD), adhesin genes (sfaS, papA, papG, papGII, papGIII, fimH, afa operon, agn43) and other virulence genes $(\operatorname{traT}, \operatorname{malX}, \mathrm{kps} M)$. The phylogenetic groups were determined by the presence of specific virulence genes [15].

\subsection{Biofilm formation assay}

Biofilm formation was quantified by culturing isolates in microtiter wells in Lysogeny Broth (LB) at $37^{\circ} \mathrm{C}$ overnight. Subsequently, $200 \mu \mathrm{l}$ of LB-medium in 96 -well flat-bottom polystyrene microtiter plates (TPK) was inoculated with $5 \mu \mathrm{l}$ (approximately $10^{5}$ bacterial strains) of the overnight culture and incubated for $24 \mathrm{~h}$ at $37^{\circ} \mathrm{C}$. The planktonic cells were then removed and rinsed three times with distilled water and stained with $0.5 \% \mathrm{CV}$ (Crystal Violet) for $5 \mathrm{~min}$. After washing with distilled water, $200 \mu \mathrm{l}$ of $95 \%$ ethanol was added, and biofilm formation was quantified at $570 \mathrm{~nm}$ using a spectrophotometric device (Tecan Trading AG, Switzerland). The strain was considered to form biofilm if an $O D \geq 1.15$ was found. This cut-off value was based on the value found for reference strain 042 known to form biofilm and used as a positive control in our study.

\subsection{Adhesion and Invasion assays}

To compare the adhesion and invasion ability (in)to urinary tract cells of the different isolates, epithelial HEK-293 cells (Human embryonic kidney cells; ATCC®LGC) were maintained in Dulbecco's modified Eagle medium (ThermoFisher Scientific, Paisley, Scotland) supplemented with $2 \%$ fetal bovine serum (VWR, Roden, The Netherlands). HEK-293 were grown in a monolayer in 24well plates and inoculated in triplicate with different isolates using a multiplicity of infection of 20. After $3 \mathrm{~h}$ or $6 \mathrm{~h}$ of incubation at $37^{\circ} \mathrm{C}$ in a $\mathrm{CO}_{2}$ incubator, cells were washed three times with phosphatebuffered saline (PBS) and then lysed using 0.1\% Triton X-100 in PBS. Lysates were collected, serially diluted, and plated onto TSA agar plates, and the number of colony-forming units was determined for each isolate. For the invasion experiment, the cells' medium was replaced after $3 \mathrm{~h}$ of incubation by a medium containing $100 \mu \mathrm{g} / \mathrm{ml}$ of kanamycin and incubated for another $1.5 \mathrm{~h}$ to kill the attached 
but not the intracellular bacteria. After incubation, cells were lysed and further processed as described above.

\subsection{Statistical analysis}

Data analysis was performed using the Statistical Package for the Social Sciences (SPSS) Software version 24.0 (SPSS Inc., Chicago, IL, USA). Statistical procedures included analysis of percentages, mean and standard deviation for categorical and numerical data. Statistical significance was assessed using the Fisher's exact test for categorical variables and the Student t-test and the Mann-Whitney U test for continuous variables. Bivariate comparisons were used to analyze the frequency of specific isolates between patients with and without risk factors. The correlation study was performed using logistic regression analysis for multivariate analysis. Variables that achieved a probability of $<0.1$ in the univariate analysis were considered for inclusion in the logistic regression models. The results with $\mathrm{p}$ values below $0.05(\mathrm{p}<0.05)$ were considered significant.

\subsection{Ethical approval and consent to participate}

This study was submitted and approved by the Pedro Ernesto University Hospital ethical committee according to Brazilian legislation and received the following CAAE number: 45780215.8.0000.5259/ All the samples used in this study were obtained from patients that signed a consent form in which they permitted the use of samples and data for this study.

\section{Conclusions}

The frequency of UTIs among female and older patients was higher than in male and younger patients. Comorbidities, surgical procedures, and previous hospitalization were the most frequently found risk factors in this study. In addition, complicated CA-UTIs, and UTIs caused by MDR/ESBL and biofilm-forming E. coli are highly frequent among hospitalized patients. Comorbidities as diabetes, neurologic and neoplastic diseases were considered to increase the risk of developing a UTI caused by MDR E. coli, and an age older than 60 years increased the risk of developing a UTI caused by ESBL-producing E. coli. No relation was found between patients having risk factors and having a UTI caused by a biofilm-forming isolate. Moreover, we did not find a statistically significant difference between the distribution of virulence and resistance genes among bacteria causing the HAUTIs, CA-UTIs, or ABU. Most bacteria isolated from patients suffering from a UTI, particularly a complicated one, belonged to high-risk E. coli lineages. UTIs caused by these highly resistant lineages were associated with a higher mortality rate. However, the susceptibility of these patients to infections is also an important factor. Finally, the combination of molecular and phenotypically characterization and epidemiological analysis of E. coli isolated from UTIs could be helpful for the risk-assessment of UTIs and for improving patient management.

Supplementary Materials: No supplementary material available.

Author Contributions: ACdC conceived, designed experiments, analyzed the results, and drafted the manuscript. NLA, JCDC, and CSS provided the samples and patient's data. MF analyzed the results and critically reviewed the manuscript. JMvD and MdTE contributed to the experiments and analyses of the results. NTM, ACdPR, AWF, MACF, and PD critically reviewed the manuscript. JWAR contributed to the experimental design, analyses of results, and drafting and reviewing the manuscript.

Funding: This research was funded by the Abel Tasman Talent Program for biomedical research talent of the University Medical Center Groningen, which aims to support high-quality international research at the University of Groningen/University Medical Center Groningen, the Netherlands. Also, this study was financed in part by the Coordenação de Aperfeiçoamento de Pessoal de Nível Superior - Brasil (CAPES) - Finance Code 001. 
Acknowledgments: We would like to thank J. A. Kuivenhoven from the Pediatrics Section of the Department of Molecular Genetics at the UMCG for providing the ATCC HEK-293 cell line used in this study.

Conflicts of Interest: The authors declare no conflict of interest

\section{References}

1. Croxen, M.A.; Finlay, B.B. Molecular mechanisms of Escherichia coli pathogenecity. Nat Rev Microbiol 2009, 8, 26-38.

2. Barber, A.E.; Norton, J.P; Spivak, A. M.; Muley, M.A. Urinary tract Infections: Current and emerging management strategies. Clin Infect Dis 2013, 57(5), 719-724.

3. Cergole-Novella, M.C.; Pignatari, A.C.C.; Guth, B.E.C.Adhesion, biofilm and genotypic characteristics of antimicrobial resistant Escherichia coli isolates. Braz J Microbiol 2015, 171, 167-171.

4. Subashchandrabose, S.; Mobley, H.L.T. Virulence and Fitness determinant of uropathogenic Escherichia coli. Microbiol Spectr 2015, 3, 1-32.

5. Neupane, S.; Pant, N.D.; Khatiwada, S.; Chaudhary, R.; Banjara, M.R. Correlation between biofilm formation and resistance toward different commonly used antibiotics along with extended spectrum beta lactamase production in uropathogenic Escherichia coli isolated from the patients suspected of urinary tract infections visit. Antimicrob Resist Infect Control 2016, 5, 1-5.

6. Eberly, A.R.; Floyd, K.A.; Beebout, C.J.; Colling, S.J.; Fitzgerald, M.J. Biofilm formation by uropathogenic Escherichia coli is favored under oxygen conditions that mimic the bladder environment. Int J Mol Sci 2017, 18, 1-12.

7. Delcaru, C.; Alexandru, I.; Podgoreanu, P.; Grosu, M.; Stavropoulos, E.; Chifiriuc, M.C.; Lazer, V. Microbial Biofilms in Urinary Tract Infections and Prostatitis: Etiology, Pathogenicity, and Combating strategies. Pathogens 2016, 5(4), 65.

8. Flores-Oropeza, M.A.; Reyes-Grajeda, J.P.; Ochoa, S.A.; Cruz-Córdova, A.; Flores-Encarnación, M.; Ramirez-Vargas, A.; Flores-Garcia J.H.; Moreno-Fierros, L., Xicohtencatl-Cortes, J. Features of urinary Escherichia coli isolated from children with complicated and uncomplicated urinary tract infections in Mexico. PLoS One 2018, 13, e0208285.

9. Campos, A.C.C..; Andrade, N.L.; Ferdous, M.; Chlebowicz, M.A.; Santos, C.C.; Correal, J.C.D.; Lo Ten Foe, J.R.; Rosa, A.C.P.; Damasco, P.V.; Friedrich, A. W.; Rossen, J.W.A. Comprehensive Molecular Characterization of Escherichia coli Isolates from Urine Samples of Hospitalized Patients in Rio de Janeiro, Brazil. Front Microbiol 2018, 9, 1-12.

10. Alves, J.R..; Pereira, A.C. M..; Souza, M.C.; Costa, S.B.; Pinto, A.S.; Mattos-Guaraldi, A.L.; Hirata-Júnior, R.; Rosa, A.C.P; Asad, L.M.B.O. Iron-limited condition modulates biofilm formation and interaction with human epithelial cells of enteroaggregative Escherichia coli (EAEC). J Appl Microbiol 2010, 108(1), 246-55.

11. Aziz, R.K.; Bartels, D.; Best, A.A.; DeJongh, M.; Disz, T.; Edwards, E.A.; Formsma, K.; Gerdes, S.; Glass, E.M.; Kubal, M.; Meyer, F.; Olsen, G.J.; Olson, R.; Osterman, L.A.; Overbeek, R.A.; McNeil, L.K.; Paarmann, D.; Paczian, T. Parrelo, B,; Pusch, D, G.; Reich, C.; Stevens R.; Vassieva, O.; Vonstein, V.; Wilke, A.; Zagnitko, O. The RAST Server: Rapid Annotations using Subsystems Technology. BMC Genomics 2008, 9, 75.

12. Ferdous, M.; Kooistra-Smid, M.A.A.; Zhou, K; P.V.; Rossen, J.W.A.; Friedrich, A. W. Virulence, Antimicrobial Resistance Properties and Phylogenetic Background of Non-H7 Enteropathogenic Escherichia coli O157. Front Microbiol 2016, 9, 1540.

13. Zankari, E.; Hasman, H.; Cosentino, S.; Vestergaard, M.; Rasmussen, S.; Lund, O.; Aarestrup, F.M.; Larsen, M.V. Identification of acquired antimicrobial resistance genes. J Antimicrob Chemother 2012, 67, 2640-2644.

14. Joensen, K.G.; Tetzschner, A.M.M.; Iguchi, A.; Aarestrup, F.M.; Scheutz, F. Rapid and easy in silico serotyping of Escherichia coli isolates by use of whole-genome sequencing data. J Clin Microbiol 2015, 53, $2410-2426$.

15. Clermont, O.; Christenson, J.K.; Denamur, E.; Gordon, D.M. The Clermont Escherichia coli phylo-typing method revisited: Improvement of specificity and detection of new phylo-groups. Environ Microbiol Rep 2013, 5, 58-65.

16. Terpstra, M.L; Geerlings, S. Urinary tract infections: how new findings create new research questions. Curr Opin Infect Dis 2016, 29, 70-72. 
17. Spoorenberg, V.; Geerlings, S.E.; Geskus, R.B.; Reijke de, T.M.; Prins, J.M.; Hulscher, M.E.J.L. Appropriate antibiotic use for patients with complicated urinary tract infections in 38 Dutch Hospital Departments: a retrospective study of variation and determinants. . BMC Infect Dis 2015, 15, 505.

18. Mazzariol, A.; Bazaj, A.; Cornaglia, G. Multi-drug-resistant Gram-negative bacteria causing urinary tract infections: a review. J Chemother 2017, 29, 2-9.

19. Yi-Hui, W.; Po-Lin, C.; Yuan-Pin, H.; Wen-Chien, K. Risk factors and clinical impact of levofloxacin or cefazolin nonsusceptibility or ESBL production among uropathogens in adults with community-onset urinary tract infections. J Microbiol Immunol Infect 2014, 47(3), 197-203.

20. Wang, A.; Nizran, P.; Malone, M.A.; Riley, T. Urinary tract infections. Prim Care - Clin Off Pract 2013, 40, 687-706.

21. Ikram, R.; Psutka, R.; Carter, A.; Priest, P. An outbreak of multi-drug resistant Escherichia coli urinary tract infection in an elderly population: A case-control study of risk factors. BMC Infect Dis 2015, 15, 1-7.

22. Tenney, J.; Hudson, N.; Alnifaidy, H.; Cheung, L.T.J.; Fung, H.K. Risk factors for acquiring multidrugresistant organisms in urinary tract infections: A systematic literature review. Saudi Pharm J 2018, 5, 678984.

23. Bardoloi, V.; Yogeesha, B. K. V. Comparative study of isolates from community-acquired and catheterassociated urinary tract infections with reference to biofilm-producing property, antibiotic sensitivity and multi-drug resistance. J Med Microbiol 2017, 66, 927-936.

24. Mathers, A.J.; Peirano, G.; Pitout, J.D.D. The role of epidemic resistance plasmids and international highrisk clones in the spread of multidrug-resistant Enterobacteriaceae. Microbiol Rev 2015, 28, 565-591.

25. Hertz, B.F.; Nielsen, J.B.; SchØnning, K.; Littauer, P.; Knudsen, J.D.; LØbner-Olesen, A.; Frimodt-MØller, N. "Population structure of Drug-Susceptible, -Resistant and ESBL-producing Escherichia coli from Community-Acquired Urinary Tract Infections". BMC Microbiol 2015, 16, 63.

26. Fusun, C.; O” zlem, K.A. P.I.; Nurtop, E.; Seref, C. I.; Layda,L.I.; Ozge Nur, A.; Yelda, C.O.O.E. The clinical impact of ST131 H30-Rx subclone in urinary tract infections due to multidrug-resistant Escherichia coli. J Glob Antimicrob Resist 2016, 4, 49-52.

27. Albaramki,J.H.; Abdelghani, T.; Dalaeen, A.; Khdair, F.; Abeer, A.; Rasha O.K.A. . Urinary tract infection caused by extended-spectrum $\beta$-lactamase-producing bacteria: Risk factors and antibiotic resistance. Pediatr Int 2019, 61, 1127-1132. 